\title{
Le salut en judo : un médium ritualisé de communication
}

Gilles Bui-Xuân et Sébastien Ruffié

\section{(2) OpenEdition \\ 1 Journals}

Édition électronique

URL : http://journals.openedition.org/corpsetculture/564

DOI : 10.4000/corpsetculture.564

ISSN : $1777-5337$

Éditeur

Association Corps et Culture

Édition imprimée

Date de publication : 1 juin 1999

ISSN : 1268-5631

Référence électronique

Gilles Bui-Xuân et Sébastien Ruffié, "Le salut en judo : un médium ritualisé de communication », Corps et culture [En ligne], Numéro 4| 1999, mis en ligne le 25 avril 2007, consulté le 10 décembre 2020.

URL : http://journals.openedition.org/corpsetculture/564 ; DOI : https://doi.org/10.4000/ corpsetculture.564

Ce document a été généré automatiquement le 10 décembre 2020.

(c) tous droits réservés 


\title{
Le salut en judo : un médium ritualisé de communication
}

\author{
Gilles Bui-Xuân et Sébastien Ruffié
}

1 Ave Caesar, morituri te salutant! C'est ainsi que les gladiateurs se présentaient à l'Empereur, avant qu'il ne donne le signal du combat. La formule est doublement exemplaire: elle a traversé le temps en tant que règle grammaticale et elle l'a transcendé comme symbole ritualisé de soumission à un destin quelquefois funeste, puisque le représentant suprême décidait du sort de ceux qui allaient risquer leur vie dans l'arène. Les combats de gladiateurs n'étaient toutefois pas des combats guerriers, n'intervenant en rien dans la conquête de territoires. Là l'affrontement était réglé, l'espace balisé, le temps compté, et l'adversaire, homme ou animal, averti de la menace explicite qui le guettait, était mis en demeure d'y répondre. Les spectateurs en étaient les témoins, et c'est leur humeur, fustigée par l'attitude du combattant, qui déterminait l'issue finale à travers « la main du Caesar ».

2 Mais des jeux au sport il n'y a qu'un pas et des Jeux du cirque aux sports de combat, le Rubicon est si vite franchi que la mort y est toujours présente, sublimée certes, autant qu'elle peut être écartée de la conscience du danger qui subsiste cependant. Car si dans l'inconscient collectif comme dans l'inconscient individuel c'est bien sa vie qui est en jeu et la mort symbolique de l'adversaire qui est recherchée ${ }^{1}$, dans la réalité de la gestion du combat il s'agit bien de « descendre » l'adversaire.

3 Le sport, espace d'expression théâtralisée par le jeu de rôles dans lequel les acteurs sportifs s'évertuent à mettre en scène les représentations de nos civilisations, est balisé par des éléments communicationnels qui permettent son approche. De la Grèce antique, où il était plongé dans l'archaïsme des croyances aux dieux de l'olympe en passant par Rome ou les Turner, puis dans nos sociétés modernes où il semble prisonnier du tout spectacle et du tout compétition, le sport reflète notre mode de pensée et d'appartenance au monde. Si de la divinité, de l'appel au sacré, un basculement séculier vers le profane s'est réalisé dans le domaine du sport, ce n'est que pour mieux caricaturer notre société. Pourtant, les cas de violence et de dopage qui l'animent encore et toujours et qui offrent tous les éléments apparents de son 
autodestruction en lui conférant sa dimension profane, préservent paradoxalement l'aura divine du champion. Il revêt encore un apparat luminescent qui le propulse dès sa victoire au rang de divinité, capable d'un seul geste d'engendrer des émotions audelà du réel, proche du mystique. Les actes codifiés, normés, répétitifs auxquels s'adonne le sportif lors de sa pratique d'élection, s'apparentent aux phénomènes rituels qui donnent au sport une essence communicationnelle. Les rituels sont en effet ces actions "répétitives, compulsives, liées à une contrainte de caractère plus promotionnel que répressif (...) ayant un caractère symbolique lié à leur fonction de communication entre les êtres par le moyen de signes standardisés » (Rivière C., 1988 : 158). Ils apparaissent comme un quasi langage. C. Javeau (1996:256-257) définit les rituels comme des «actes codifiés, se produisant lors des mêmes occasions, et présentant un rapport à un objet sacralisé, en l'occurrence le lien qui unit, avec plus ou moins de profondeur, deux (ou plusieurs) personnes, et qui est comme le symbole de la cohésion sociale en général ». Le sport par son rapprochement au sacré, son inscription dans une temporalité et ses formules d'intronisation tissant le lien social, révèle toute sa dimension rituelle. Or même si le sport se détache du magico-religieux, condition rituelle de la constitution d'un rituel, C. Rivière (1995) rappelle que dans le quotidien, «le profane peut revêtir un sens sacré sans que les actes en soient gérés par une instance religieuse », et M. Augé (1998) parle de "sacralité laïque », considérant l'objet sport comme porteur d'une ritualité moderne. Les stades, comme les églises pour la religion, dépassent leur vocation d'accueil physique des personnes pour accomplir un rituel, producteur de moments récursifs. Bourdieu, quant à lui décrit l'institutionnalisation effective que produit le rite, comme étant sa fonction primaire. Il $\mathrm{y}$ a dans le sport, ou plus exactement dans les pratiques périphériques du sport, une dimension rituelle institutionnalisante qui s'exerce pour amplifier et consacrer l'objet de ces rites. Cette fonction d'institutionnalisation du rituel demeure, non pour renforcer la position de l'objet, mais pour séparer d'une manière intangible le sportif et le non sportif, le champion et le simple pratiquant, « Séparer ceux qui l'ont subi non de ceux qui ne l'ont pas encore subi, mais de ceux qui ne le subiront en aucune façon et d'instituer ainsi une différence durable. [...] Il y a par la même, un ensemble caché par rapport auquel se définit le groupe institué » (Bourdieu P., 1982 : 122). Les cérémonies officielles et officieuses à l'égard des champions du monde de football sont les témoignages explicites d'une institutionnalisation du mythe visant sa sacralisation.

4 Ainsi le sport s'élabore par et à travers les rites et les rituels qui le composent. Cependant, l'objet ici ne réside pas dans la connaissance de ce phénomène déjà bien repéré, mais plutôt dans la construction du sens que les acteurs effectuent dans leur confrontation progressive au rite. L'attention se porte ici sur ce qui semble être un micro-rituel d'interaction explicite dans le domaine du sport, le salut dans une discipline emprunte de connotations symboliques : le judo.

Que signifie le salut, micro-rituel d'interaction, pour les acteurs de la discipline? Quels sens construits dans l'interaction? Quelles communications s'établissent dans le geste simple et normé entre et pour les judokas? Ce sont ces questions qui guident cette démarche qui envisage plusieurs points. En premier lieu, pour écarter toute dissonance, il nous convient de s'interroger sur la nature de l'objet dans sa fonction de micro-rituel. Ensuite une conception capable d'appréhender à la fois la construction d'un sens et sa valeur communicationnelle sera avancée. Ainsi une méthode théorique, extraite de procédures de communication constructivistes et systémiques est posée. Enfin, un 
traitement de données recueillies grâce à un questionnaire proposé aux acteurs du monde du judo viendra appuyer les hypothèses

Le salut en judo comme microrituel

6 Si chaque société fonde son identité à travers "Un Rituel», si celui-ci s'exprime primitivement dans des pratiques magiques, puis religieuses, pas seulement cérémonielles mais s'inscrivant dans les actes parfois les plus bénins de la vie quotidienne, si notre époque connaît un déficit de spiritualité propre à l'avènement des sociétés modernes, alors le sport n'est-il pas en train de se substituer à la communion des hommes dans le sacré, pour développer à l'échelle planétaire une humanité susceptible de se reconnaître dans des rites communs, assimilables par tous?

Dans ces conditions, un sport comme le judo, et plus particulièrement le salut qui lui est inhérent participe-t-il, en tant que microrituel, à la ritualisation de la société avant même de participer à la ritualisation de l'activité ? La dimension sociale du spectacle sportif, auréolée par une volonté médiatique et superficielle, si elle se donne à voir comme une spectacularisation à outrance inadéquate avec les valeurs religieuses propres aux rites originaires, semble, dans un parallèle, se juxtaposer à ce cadre, pour incarner une certaine visée dogmatique. Pour J.M. Brohm, les analogies métaphoriques à la religion sont abondantes dans la pratique sportive qui reproduit «objectivement certaines structures du mythe, du sacré, du mystique, du culturel, du rituel » (Brohm J.M., 1983 : 108). Le cérémonial, la temporalité, le kimono, le tatami, le salut, voire les « imprécations », sont autant d'éléments classificatoires du judo en tant qu'acte rituel. Pourtant une controverse pèse sur les aspects ritualisés du sport considérés par certains comme de simples médiateurs dont la vertu ne réside que dans la réalisation du spectacle programmé, sans qu'ils ne remplissent leurs fonctions sociales. Mais si «le rituel échappe dans sa signification transcendante, à une totalité profane du monde sportif " (Pruneau J., 1998: 7), l'expression rituelle des arts martiaux, de laquelle émerge la recherche originelle "d'un mouvement fondamental et fondateur de la relation au monde » (Baudry P., 1992 : 157) constitue une exception. Patrick Baudry s'interroge : « les arts martiaux et les sports de combat sont peut-être des manières de vivre ce qui fascine; de passer de la consommation passive d'images à l'échange, du spectacle au rituel. » (Baudry P., 1984 : 68-73) Le judo participerait donc à un ensemble de pratiques rituelles. Le salut, dans sa forme répétée, visant plus que symboliquement à produire "les critères d'un caractère vertueux, d'une conduite honorable, de conduites sociales justes» (Walzer M., 1990: 62), n'est-il pas le fruit, la traduction technique ${ }^{2}$, du souci, souligné par E. Goffman, de manifester une bonne «maittrise des impressions " en réponse aux attentes d'autrui? Le salut se constitue comme une interaction sociale normée dans une pratique ritualisée, visant à établir un lien social entre les individus : "Balise de la mémoire, les microrituels du quotidien perpétuent une identité dans la mesure où leur pratique révèle l'identification à un groupe spécifique et différencié. Ils permettent, selon E. Goffman, de rester honorable et porteur d'honneur " (Rivière C., 1991: 239). Le terme microrituel étant défini comme « ces expressions brèves et superficielles qui accompagnent incidemment (...) les actions quotidiennes » (Goffman E., $1988: 213$ ) et que Javeau nomme des «microrituels du quotidien» (Javeau C., 1992: 60). Le terme quotidien étant ici superflu (car la pratique peut se révéler en dehors du quotidien), il n'en reste pas moins que le salut apparaît, dans une interaction de prime abord duelle, comme un "rituel de contact " (Goffman E., $1988: 213$ ). 
Les fonctions sociales du salut en judo

8 Mais le rituel du salut ne se contente pas de signifier quelque chose, il agit également sur ceux qui le pratiquent au point où ils ne sauraient s'en dispenser. Une hypothèse peut alors être formulée : le rituel du salut a toujours eu pour fonction de mettre les combattants en "consonance socio-conative ${ }^{3}$ pour que le combat puisse avoir lieu.

9 Le salut, la forme auguste qu'il incarne et qu'il entend dénoter, renvoie très explicitement à la notion de respect et d'honorabilité. L'honneur dans la pratique du judo et dans l'ensemble des arts martiaux, l'inclinaison métaphorique, l'humilité qui est produite dans le geste du salut, l'ensemble des expressions de cette valeur morale peut être abordé comme un "ritème» de présentation indiquant la sacralité des partenaires, mais aussi celle de leurs relations. Cette ritualité marque simultanément les interdits attachés à la personne comme au combat. La redondance du geste, sa « routinisation » (Giddens A., $1987: 109$ ), vient signifier, comme des balises codifiées à l'intention de la mémoire, que la pratique de cette discipline ne peut s'effectuer en dehors de ce contexte culturel. La procédure mise en place vise à la conservation de la valeur d'honorabilité portée par l'acteur et par les normes culturelles du judo, dans un processus qui l'actualise à chaque protocole. Par analogie aux "parlures vacantes " illocutoires ou non, décrites par C. Javeau, d'autres fonctionnalités se manifestent ici. Le salut en judo peut, comme le bonjour d'un signe de la main, revêtir une fonction phatique. Il existe alors uniquement pour maintenir ou établir le contact entre les protagonistes. Il veut signifier, sous une forme gestuelle, son existence au monde et donc à autrui. La dimension standardisée décrite par certains combattants dans sa production, vient conforter cet aspect. Mais réduire ainsi sa fonction serait plus qu'une simplification univoque d'un geste qui semble vouloir en dire plus long. Car à l'opposé de ces formules vacantes, le salut s'il n'est pas illocutoire, propose une communication implicite et explicite pour les judokas. Le lien social, représenté par le salut, qui réunit les partenaires, suggère une fonction de rapprochement des acteurs dans une unification sociale. La salutation sert de présentation à autrui, confirme son existence au monde et l'appartenance communautaire au groupe institué. Mais jouant un rôle, incorporé dans la société des judokas, se découvre simultanément une «distance au rôle » pour l'acteur.

Il y a tout d'abord une distance physique abordable, pour le spectateur, dès le premier instant. En effet si, comme l'explique C. Javeau (1996), l'acte de serrer la main à un individu, comme en lutte ou en sambo, peut signifier, par le bras tendu, une protection de l'espace intime, donc une mise à distance d'autrui pour éviter tout contact indésirable, que peut-on dire d'un salut où les corps ne s'effleurent en aucune manière, comme en judo, karaté, aïkido? Ce salut lointain va signifier à autrui qu'une ligne intangible s'instaure, protégeant ainsi sa personne, et que tout franchissement de cette limite peut entraîner des désagréments pour l'autre. Car si le salut marque un espace provisoirement protégé, 4 mètres, 2 tatamis, correspondant aux 6 pas du salut militaire, il marque également un temps protégé, un avant et un après. Dans des disciplines de combat, comme la boxe, l'artifice faisant office de salut est la rencontre des deux poings. La prise de contact est physique et c'est dans l'affirmation de son corps, de sa puissance, que les boxeurs se proposent l'un à l'autre. Croiser les armes, le fer en escrime, le boken en kendo, permet également de tester la volonté de l'autre d'entrer dans l'affrontement. Dans le cas du judo, la symbolique est bien plus marquée 
par l'espace créé. Il est intéressant de souligner que l'irrespect de la limite instituée s'opère objectivement entraînant le combat dont la mort symbolique est la résultante ${ }^{4}$, ce qui est impossible dans la vie courante. Au-delà de cette distance physique, le rituel facilite, quand ce n'est pas sa fonction principale, une distance " instaurée par l'acteur entre son "moi profond" et son "moi projeté" (sur autrui), dans une ambiance de mensonge social généralisé » (Javeau C., $1996: 260)^{5}$. Si cette notion de distance au rôle, chère à E. Goffman, n'était pas respectée, alors le combat codifié ne pourrait plus suivre la convenance normative à laquelle les protagonistes sont tenus : il ne pourrait sans doute même pas avoir lieu. Le salut est soumis à une corrélation culturelle riche qui unit et agrège les participants. Il fait appel à une mémoire collective des acteurs tout en instituant une hypocrisie généralisée (car le respect ou l'honorabilité, peuvent n'être qu'une façade), pour maintenir l'identique dans le temps. Il est le marqueur du temps social qui se joue. Au-delà de l'aspect pratique d'ouverture et de fermeture, il montre la durabilité du groupe. Il permet la continuité. Si le salut n'était pas maintenu, il se produirait alors une rupture temporelle qui générerait l'insécurité et par là, la violence $^{6}$. À l'instar de C. Kerbrat ${ }^{7}$, s'affirme ici une des fonctions fondamentales du rite qui se forge dans un sécuritarisme prégnant. Il y a une sorte de mimétisme visant à reproduire la même activité, sous les mêmes codes, par le temps passé, pour le temps futur, mais hic et nunc. Le ritème du salut vient donc, dans son ensemble, inscrire la sécurité dans la temporalité, en tissant un lien social unificateur qui « ratifie » (pour reprendre un terme de E.Goffman) les acteurs considérés dans leur conscience individuelle et collective d'être au monde. Si la violence est ainsi régulée, elle n'est toutefois pas prête de disparaitre, ni dans le sens ni dans les gestes. Ce qui fait dire à Patrick Baudry : «Si l'on veut bien comprendre que la violence accompagne l'existence sociale, qu'elle en est constitutive, autrement dit qu'elle ne résulte pas seulement d'une organisation inégalitaire ou d'une exploitation politique, on peut alors admettre qu'il vaut mieux composer avec elle que de songer à sa disparition » (Baudry P., 1984 : 71).

11 Enfin, l'introduction que signifie l'acte pratique du rite, le passage dans et hors $d u$ combat, n'est pas sans rappeler les rites de passages étudiés par Van Gennep. Cependant, si les phases successives du passage, normées et institutionnalisées, standardisées par les notions de "séparation, marge, agrégation » (Van Gennep A., 1909), se révèlent comme une caractéristique des rites de passage, il nous semble qu'ici, si elles existent comme telles, elles le sont dans une certaine intemporalité ou à l'opposé dans un temps réduit à l'instant. La symbolique du passage peut être diffuse à tout le rituel du combat, comme elle peut se signifier simplement dans l'acte du salut. Il y a en effet de la séparation dans la situation de face à face, comme les combattants peuvent tout aussi bien être séparés de leur communauté, ou mis en marge lorsqu'ils sont sur le tatami. En fonction du contexte de référence peut apparaître simultanément à cette notion de marge, l'agrégation des deux acteurs dans la "communitas", considérée par Turner (1990) comme la marge, mais aussi l'agrégation des combattants aux valeurs propres à la discipline, à la communauté.

Mais ce qui semble plus fondamentalement marquer tous les rituels de salut, dans l'histoire et dans les modalités diversifiées du combat, c'est bien son rapport à la mort, vécu symboliquement ou comme la mise en jeu réelle de sa vie. Si, selon Durkheim, "les rites sont des règles de conduite qui prescrivent comment l'homme doit se comporter avec les choses sacrées " (Durkheim E., 1912) comment ne pas admettre que ce qui se joue dans le combat puisse être sacré, et comment l'aborder sans faire appel aux rites? Il est alors possible de revenir à la notion de «life crisis », développée par 
A. Van Gennep, en considérant que la mise en jeu de sa vie, même symbolique, même répétitive, appelle un rite de passage dont le salut constituerait le seuil de liminarité. D'ailleurs, plus le niveau est élevé, plus les combattants suivent les phases décrites par Van Gennep. La séparation est effective quand le combattant s'isole pour se concentrer ${ }^{8}$ ; la phase liminaire serait circonscrite au salut ; la réagrégation à la communauté des combattants actifs ne se jouant que le temps du combat. Mais il se peut que le combat, entouré de son rituel, représente l'épreuve à laquelle le judoka serait soumis avant de pouvoir intégrer la communauté des vrais combattants.

Il reste à définir les modèles théoriques qui permettraient de cerner les contextes dans lesquels s'inscrit cette démarche.

La construction d'un sens partagé

Cette étude sur le microrituel du salut en judo a une visée compréhensive : comprendre quels sens les acteurs du judo construisent autour de ce ritème en est l'objectif. Sens partagé ou sens divergent ? En s'arrêtant sur ce premier point, il est possible, au regard de la fonction primordiale des rituels, celle de la constitution d'un lien social, d'opter pour la construction d'un sens partagé. Mais comment appréhender cette construction? Comment la rendre éminemment explicite ? La communication apporte des éléments capables d'aborder ce phénomène. Les méthodes basées sur le modèle systémique et constructiviste de la communication «se centrent sur un [...] enjeu fondamental de la communication : le partage de sens » (Mucchielli, 1994 : 25). Le rituel du salut peut être envisagé comme une communication ; c'est une activité partagée par les acteurs, d'où émerge, pendant son déroulement, la construction de significations communes qui servent de référence pour la suite des échanges et des actions. Le salut s'élabore dans la perspective commune qui tend à la mise en œuvre d'intentions, ici la présentation, le respect, l'introduction ou la fin du combat, en les rendant manifestes. Il fait appel à des référents collectifs qui doivent être réfléchis dans la situation donnée, car c'est « la mise en relation des éléments communicationnels avec la situation » (ibid. p.15) qui donne le sens. La situation vient en appui du salut, celui-ci ne peut être compris que dans les contextes dans lesquels les acteurs le font intervenir : «Le sens émerge de configurations situationnelles dans lesquelles les activités se déroulent et qui sont co-construites par les acteurs en présence» (ibid. p.22). Ce modèle contextuel demande, comme le souligne P. Watszlawick (1972), un positionnement particulier de l'observateur. La situation d'étude doit nécessairement bénéficier d'un cadrage large faisant apparaître ledit phénomène. Ce cadrage large doit être appréhendé, dans un souci d'élucider le sens commun des acteurs, à travers différents contextes communicationnels. Le contexte spatial, le lieu de la pratique, le contexte physique et sensoriel qui fait percevoir l'acte, la contextualisation temporelle, dont l'importance pour le rite d'une nécessaire inscription dans le temps a été décrite, le positionnement et la structuration des relations autour de l'activité, l'appel à des normes culturelles collectivement partagées par la communauté judokate, la construction de référents relationnels ou de la qualité des relations dont le salut vient signifier les règles et l'expression identitaire en tant que combattant, sont autant de contextes qui permettent de réfléchir autour d'un cadrage large et pertinent, pour comprendre la construction du sens dans la conduite des acteurs. Au regard de l'hypothèse, à savoir la mise en consonance socio-conative des combattants pour que le combat puisse se dérouler, l'importance donnée aux différents contextes permet la mise en évidence du sens et donc, peut-être, d'une référence commune, généralisée et vécue par l'ensemble des acteurs. L'intentionnalité des acteurs va donc se comprendre dans un champ 
épistémologique comprenant une vision appropriée du rituel en tant que communication. Les questionnaires, constituant notre méthode d'investigation des représentations régissant l'acte du salut en tant qu'élément communicationnel, vont permettre de tester, à travers les contextes considérés, si le sens pris par le salut apparaît, dans une commune mesure, comme une convergence sémiotique, de l'interprétation des acteurs.

Aspects méthodologiques

Les aspects présentés plus haut s'appuient sur une longue expérience des tatamis, et sur un recueil d'entretiens auprès d'un panel de judokas d'origines diverses. Leur analyse thématique permet de dégager des lignes de force qui peuvent être résumées ainsi :

- le salut ferait partie de l'étiquette du judo. Il exprimerait une forme de politesse, de savoirvivre.

- comme cérémonial, le salut serait un rituel qui résumerait toute la symbolique du judo.

- il signifierait que l'on serait prêt à respecter les règles élémentaires du combat, l'adversaire et l'issue de la rencontre.

- il serait nécessaire pour canaliser la violence qui risquerait de s'exprimer.

Bien que l'hypothèse de la mise en consonance socio-conative puisse déjà se vérifier à travers ces thèmes, notre connaissance des sports de combat amenait à formuler des thèmes complémentaires, tels que :

- le rituel du salut se retrouve sous une forme ou une autre dans tous les sports de combat et tous les arts martiaux.

- il pourrait avoir une fonction de conjuration de l'angoisse face à la mise en jeu de sa vie.

- c'est pourquoi l'expérience du combat, la pratique des tatamis, le grade, seraient des éléments de différenciation du rapport au salut.

- voire, entre les instances officielles, fédérales, qui prôneraient une véritable éthique de l'activité, et les judokas ordinaires qui pourraient l'effectuer de façon machinale, il pourrait y avoir la distance de la théorie à la pratique.

17 C'est pourquoi ces décalages de représentations ont été l'objet d'une vérification, un bref questionnaire étant soumis à différentes populations de judokas: professeurs expérimentés engagés dans la vie fédérale, jeunes professeurs en stage de formation optionnelle, judokas de clubs.

Le questionnaire est composé de 13 questions fermées et d'une seule question ouverte : "Que pensez-vous vous même du salut en judo?»

Les résultats des questions fermées peuvent être globalement présentés ainsi :

\begin{tabular}{|l|l|l|}
\hline & Modalité citée en ${ }^{\circ} 1$ & Modalité la moins citée \\
\hline \hline 1. Histoire & oui $(65=92,86 \%)$ & non $(4=5,71 \%)$ \\
\hline \hline 2. Sports de combat & oui $(55=\mathbf{7 8 , 5 7 \% )}$ & non $(\mathbf{1 5}=\mathbf{2 1 , 4 3 \% )}$ \\
\hline \hline 3. Symbole & oui $(63=90,00 \%)$ & non $(7=10,00 \%)$ \\
\hline \hline 4. Étiquette & oui $(67=95,71 \%)$ & non $(3=4,29 \%)$ \\
\hline
\end{tabular}




\begin{tabular}{|l|l|l|}
\hline 5. Politesse & oui $(60=85,71 \%)$ & non $(9=12,86 \%)$ \\
\hline \hline 6. Angoisse & non $(44=\mathbf{6 2 , 8 6 \%})$ & non $(\mathbf{2 4}=\mathbf{3 4 , 2 9 \% )}$ \\
\hline \hline 7. Partenaire-adversaire & oui $(49=70,00 \%)$ & non $(21=30,00 \%)$ \\
\hline \hline 8. Vérité & non $(40=57,14 \%)$ & oui $(28=40,00 \%)$ \\
\hline \hline 9. Refus & non $(59=84,29 \%)$ & oui $(10=14,29 \%)$ \\
\hline \hline 10. Compétiteur & oui $(56=80,00 \%)$ & non $(13=18,57 \%)$ \\
\hline \hline 11. Professeur & non $(40=57,14 \%)$ & oui $(30=42,86 \%)$ \\
\hline \hline 12. Handicapés & oui $(59=\mathbf{8 4 , 2 9 \% )}$ & non $(2=\mathbf{2 , 8 6 \% )}$ \\
\hline
\end{tabular}

Tableau 1 : résumé des résultats de la population totale (70 observations)

Il est à noter qu'une question porte sur la possibilité des personnes handicapées mentales de donner un sens au salut. L'opportunité de faire passer le questionnaire lors d'un stage international organisé par la Commission nationale «judo et personnes handicapées » de la Fédération française de judo en a été l'occasion. Cette question permettait toutefois de mesurer la part dévolue au cognitif dans le salut, ou la « croyance » en des vertus plus « primaires».

L'ensemble de la population présente une grande homogénéité dans ses réponses, sauf pour 3 items qui sont : l'angoisse, la vérité et les professeurs, sur lesquels les avis sont partagés, et qui correspondent aux questions :

- «Le salut permet-il de juguler l'angoisse?»

• «Considérez-vous le combat comme l'épreuve de vérité du judo ?»

• «Les professeurs accordent-ils la même importance au salut que leurs élèves ?»

Ces premières constatations viennent renforcer l'idée de dépendance aux contextes situationnels. En effet, à la lumière de cette théorie de la communication, il apparaît que dans des contextes communs à tous les judokas, combattants, professeurs expérimentés ou novices, peu de différences significatives se manifestent. Le salut représente pour chacun d'entre eux des valeurs de respect, de code moral, d'honorabilité inhérentes à la valeur symbolique de l'activité. L'appartenance à la communauté des judokas se fonde sur ces mêmes références normatives et culturelles. Elles sont totalement incorporées et participent d'un point de vue contextuel à une vision commune du monde. En revanche des dissonances apparaissent lors de situations dans lesquelles l'appréhension contextuelle diffère suivant l'âge, l'expérience ou la fonction dans le monde du judo. Pour ce qui est du combat, où deux contextes s'affrontent, celui de l'entraîneur et celui du combattant, la vision de l'activité ne sera plus là même. Que le salut puisse juguler l'angoisse, ou que le combat soit l'épreuve de vérité en judo, dépend expressément de la situation dans laquelle se trouvent les interviewés. S'ils sont combattants, ils vont développer une vision sujette à leur contexte de référence qui sera différente de celle des entraîneurs. 
Et cependant la comparaison des résultats des professeurs expérimentés et des professeurs novices ne laisse apparaître aucune différence significative (chi2 $=18,12$, $\mathrm{ddl}=28,1-\mathrm{p}=7,70 \%$ ). Il en est d'ailleurs de même entre les professeurs novices et les judokas de club (la dépendance n'est pas significative $[$ chi2 $=16,84, \mathrm{ddl}=23,1-\mathrm{p}=$ $18,28 \%]$ ). Pourtant, la confrontation des trois populations sur l'ensemble du questionnaire témoigne d'une différence. La dépendance est certes peu significative (chi2 $=33,46, \mathrm{ddl}=46,1-\mathrm{p}>95,00 \%$ ), mais elle semble toutefois révéler que le temps passé sur les tatamis a permis à ces enseignants d'incorporer d'autres valeurs, qui ne relèvent pas de la simple transmission de connaissances, mais d'une assimilation progressive des constituants les plus anodins de la discipline.

Les questions qui sont à l'origine de cette différenciation sont :

- «Retrouve-t-on le salut dans tous les sports de combat?»

- «Les personnes handicapées mentales peuvent-elles percevoir le sens du salut en judo?»

Tableau 2 : croisement des questions 14 (statut) et 2 (sports de combat)

- À quel titre êtes-vous présent à ce stage?

- Retrouve-t-on le salut dans tous les sports de combat?

\begin{tabular}{|l|l|l|l|}
\hline Statut / Combat & Oui & Non & TOTAL \\
\hline \hline Stagiaire & 13 & 2 & 15 \\
\hline \hline Délégué & 19 & 11 & 30 \\
\hline \hline Club & 23 & 2 & 25 \\
\hline \hline TOTAL & 55 & 15 & 70 \\
\hline
\end{tabular}

(chi2 $=7,40, \mathrm{ddl}=2,1 \mathrm{p}=97,53 \%$ )

La dépendance est peu significative

24 Les résultats de cette question semblent paradoxaux. En effet, une part importante des professeurs expérimentés répondent "non » à l'hypothèse de l'universalité du salut dans les sports de combat, quand pratiquement tous les stagiaires et judokas ordinaires semblent y croire fermement. On est donc amené à penser qu'il ne s'agit pas de contester la présence effective d'une pratique quasi universelle dans ce domaine, mais plutôt le sens qu'ils lui rattachent, et que seule l'expérience permet d'intégrer. Ce sens ne se construirait donc pas dans l'observation, ni dans la rationalité, mais dans le « ressenti » et son pouvoir différenciateur. Le registre de la conation s'affirme bien ici, et non plus celui de la cognition. Si le salut en judo se ritualise, c'est parce qu'il touche, comme quelques autres arts martiaux, à l'irrationnel, au sacré, à la subjectivité transcendantale de ceux « qui ont trouvé la voie».

Tableau 3 : croisement des questions 14 (statut) et 12 (handicapés)

- À quel titre êtes-vous présent à ce stage ?

- Les personnes handicapées mentales peuvent-elles percevoir le sens du salut en judo?

\begin{tabular}{|l|l|l|l|l|l|l|l|l|l|l|l|l|l}
\hline Handicapés / Statut Non réponse & Oui & Non & TOTAL \\
\hline
\end{tabular} 


\begin{tabular}{|l|l|l|l|l|}
\hline Stagiaire & 0 & 15 & 0 & 15 \\
\hline \hline Délégué & 0 & 28 & 2 & 30 \\
\hline \hline Club & 9 & 16 & 0 & 25 \\
\hline \hline TOTAL & 9 & 59 & 2 & 70 \\
\hline
\end{tabular}

(chi2 = 20,82, $d d l=4,1 p=99,97 \%$ )

La dépendance est très significative

En dehors de la conjoncture de passage du questionnaire, la question fait ressortir non seulement l'expérience du handicap, mais toutes les projections et croyances qu'il inspire. Ainsi, le nombre important de non réponses de la part des judokas de clubs les positionnent irrémédiablement dans une démarche cognitiviste, la question étant de savoir si un handicap mental profond permet tout de même de comprendre ce que saluer veut dire. Les enseignants, eux, ne se posent pas cette question, probablement parce qu'ils sont persuadés que le salut ni ne s'apprend ni ne se comprend, mais qu'il s'incorpore, et que la déficience mentale n'est pas une entrave au procès d'incorporation.

C'est finalement cette distance progressive à une rationalité triomphante au profit d'une intersubjectivité qui peut se dispenser de raison, qui permet de percevoir comment s'installent les représentations des professeurs, et leurs usages. Ce sont les plus nombreux à penser que le salut peut juguler l'angoisse (question 6).

À la différence des judokas de clubs qui saluent leur adversaire un peu par obligation, la plupart des professeurs attribuent le même sens au salut d'un partenaire qu'à celui d'un adversaire (question 7).

On retrouve la même tendance en ce qui concerne le sens que pourrait donner au salut un compétiteur et un non compétiteur (question 10).

Enfin, les professeurs savent bien, eux, qu'ils n'accordent pas le même sens au salut que leurs élèves, même si ces élèves le croient (question 11).

31 Un «habitus » du salut s'installe peu à peu, véritable système de dispositions et de perceptions, générateur de pratiques. Disposé à combattre, percevant que l'adversaire est également disposé à respecter les règles, alors l'affrontement codifié devient possible. Le salut, dans ces conditions, est bien ce médium de communication sans lequel il faudrait déployer l'explicite quand l'implicite suffit à engager l'action.

Les résultats du questionnaire permettent de préciser les représentations sur lesquelles repose cet implicite commun :

- Les enseignants comme leurs élèves ne contestent pas l'atemporalité du salut. Il semble avoir toujours existé.

- D'ailleurs le salut se retrouve dans la gestuelle de tous les sports de combat.

- En effet il représente un symbole.

- Ce qui pourrait correspondre à une formule de politesse dans la vie courante.

- D'ailleurs il faudrait tendre à ne pas faire de différence entre le salut qui précède le combat contre un adversaire, de celui qui précède le travail avec un partenaire, et qui devrait revêtir le même sens. 
- Ainsi le refus du salut serait incompatible avec la pratique du judo.

- Quelle que soit d'ailleurs la modalité de pratique car le non compétiteur peut fort bien donner le même sens au salut que le compétiteur.

- Ce sens, les personnes handicapées mentales peuvent aussi parfaitement le percevoir. du salut en judo, elle a permis de dresser un tableau des termes les plus utilisés.

Tableau 4 : résultats de la question ouverte

\begin{tabular}{|l|l|l|l|l|l|l|l|}
\hline & $\begin{array}{l}\text { Non- } \\
\text { réponses }\end{array}$ & $\begin{array}{l}\text { Nombre } \\
\text { de mots }\end{array}$ & $\begin{array}{l}\text { Nombre } \\
\text { moyen de } \\
\text { mots }\end{array}$ & $\begin{array}{l}\text { Nombre de } \\
\text { mots } \\
\text { différents }\end{array}$ & $\begin{array}{l}\text { Nombre } \\
\text { d'hapax }\end{array}$ & $\begin{array}{l}\text { Fréquence } \\
\text { maximum }\end{array}$ & $\begin{array}{l}\text { Mot le } \\
\text { plus } \\
\text { fréquent }\end{array}$ \\
\hline $\begin{array}{l}13 . \\
\text { perso }\end{array}$ & 4 & 1532 & 23,21 & 336 & 254 & 48 & respect \\
\hline
\end{tabular}

Mots les plus fréquents pour chaque question:

Question 13. (perso) : respect (48) ; salut (35) ; judo (25) ; partenaire (14) ; adversaire (13) ; début (12) ; combat (12); marque (11); règles (10); partie (10); fin (10); pratique (9); important (9); activité (8); vis (8) ; sens (8) ; politesse (8); fait (8); autrui (7); Valeur (7) ; forme (6); code (6) ; temps (6) ; base (5) ; symbole (5) ; élèves (4); moral (4) ; acceptation (4); compétition (4) ; Permet (4).

Le terme de « respect » vient largement en tête. Ce respect concernerait aussi bien les « règles" que les "partenaires ou adversaires ». Les règles sont bien sûr celles du combat, mais aussi celles de l'acceptation pour soi d'un engagement dans une épreuve. La notion de partenaire ou d'adversaire s'adresse moins au statut qu'au rôle de tout judoka, professeur, élève, et par extension autrui. Le «temps » est également évoqué, le salut marquant le début et la fin d'un cours, d'un travail avec un partenaire, d'un combat. C'est un moment important qui donne non seulement le départ, mais qui exprime aussi un retour. Le salut est enfin "sens, signe, signification». Il contient toutes les « valeurs » du « code moral », il est un « symbole » de « loyauté ».

Aucune véritable distinction n'a pu être relevée entre les trois groupes. Le terme de « règles » est le seul qui, paradoxalement, revient bien moins souvent dans le groupe des judokas de clubs, pourtant tous compétiteurs. Comme si cela allait de soi...

Conclusion : un sens particulièrement partagé

Le judo a réussi à régler la violence originelle de l'homme en établissant un glissement progressif... de la parole au geste, du célèbre de morituri te salutant à une simple inclinaison du buste. Ce geste rituel contient tout le judo, que son fondateur Jigoro Kano résumait en une devise: Jita kyoei, autrement dit «entraide et prospérité mutuelle!»

Ainsi l'inclinaison du salut exprime l'inclination à agir ensemble pour s'élever communément. Le rapport au divin qui aurait pu être révélé dans une croyance ordalique, lors d'une épreuve, n'a pu être établi. Le salut n'est donc ni figure magique, ni incantation, qui aurait pour fonction de conjurer son propre sort, individuellement, mais plutôt inscription dans une véritable éthique d'une humanité qui progresse. Ce qui ne signifie pas qu'il puisse se dispenser de sacré. Au contraire, sa propension à être lui-même sacralisé, il le doit à la tradition judoïstique qui, en établissant son rituel comme tel, l'a fondé en respectabilité. Le salut inspire le respect parce qu'il incite au 
respect. Il contribue ainsi à l'incorporation d'un ordre moral et social, comme élément constitutif primordial de cet ordre : le judo. Les professeurs les plus expérimentés l'ont bien compris, n'acceptant aucune concession sur le salut, que les compétiteurs ou certains professeurs novices auraient tendance à négliger.

La question a été posée de savoir si le salut pouvait avoir une fonction cathartique, en réaffirmant ce qu'il est difficile d'admettre : le combat peut conduire à la mort et il faut accepter son sort ${ }^{9}$ Mais il est apparu que contrairement à celui des gladiateurs, le salut en judo n'est pas asymétrique. Il est code partagé, qui exprime clairement que les règles de conduite, le code moral, sont également partagés. Il est abrégé d'une négociation, médium d'une communication établie à l'avance. C'est alors que la prospérité peut devenir mutuelle, et le salut symbole, en réprimant l'individuel pour toucher l'universel.

Mais ce sens partagé et la situation de communication sont étroitement liés. Le salut n'a de valeur que dans certains contextes, qu'il éclaire en retour, voire qu'il provoque. Ainsi le salut d'invitation à faire un randori (c'est-à-dire un combat d'entraînement) ensemble ne saurait être assimilé à l'inclinaison d'un prétendant invitant une Catherinette à faire un tour de piste. L'un cherche à séduire ou à " conquérir " quand l'autre cherche la voie d'un progrès commun.

Mais avant d'en arriver là le chemin est-il long et difficile ? Ou au contraire le rituel estil aussi simple que limité, tant dans sa gestuelle que dans sa signification, qu'il n'exigerait pas une complexification progressive et expérientielle? Il est clairement apparu que le temps en influençait le sens. C'est que le rituel permet de vivre l'instant à l'unisson, chacun se chargeant de lui donner un sens particulier, et que le salut en judo représente donc bien ce médium de communication élémentaire qui permet d'établir la consonance socio-conative sans laquelle l'activité elle-même serait impossible.

\section{BIBLIOGRAPHIE}

Augé M. (1998) Un sport ou un rituel ?, Manière de voir le monde diplomatique, 39, 74-78.

Baudry P. (1984) Questions sur la violence ludique, Quel Corps ?, 68-73.

Baudry P. (1992) La ritualité dans les arts martiaux, Cahiers internationaux de sociologie, XCII, 143-161.

Bourdieu P. (1982) Ce que parler veut dire : L'économie des échanges linguistiques, Paris, Librairie Arthème Fayard, 122-125.

Brohm J.M. (1983) La religion sportive. Éléments d'analyse des faits religieux dans la pratique sportive. Actions et recherches sociales. Idéologies, magies, et religions, 3, (Nouvelle série vol.12), 101-117.

Giddens A. (1987) La Constitution de la société, trad. Franç., Paris, PUF, v. chap. 2.

Goffman E. (1988) Les Moments et leurs hommes, textes recueillis et présentés par Winkin Y., Paris, Seuil/Minuit. 
Javeau C. (1992) Microrituels et gestion du temps, Cahiers internationaux de sociologie, Vol. XCII, 59-71.

Javeau C. (1996) Parler pour ne rien dire, La ritualisation au quotidien, Ethnologie française, XXVI, $2,255-263$.

Mucchielli A., Corbalan J.A., Ferrandez V. (1998) Théorie des processus de la communication, Paris, Armand Colin.

Pruneau J. (1998) Rites et rituels dans le sport : évolution et transformations dans la pratique traditionnelle de la joute languedocienne, Mémoire de D.E.A. STAPS, Bromberger C. (dir.), Université de Montpellier 1.

Rivière C. (1988) Les Liturgies politiques, Paris, PUF.

Rivière C. (1995) Les Rites profanes, Paris, PUF.

Walzer M. (1990) Critique et sens commun, trad. franç., Paris, AGALMA/La Découverte.

Watzlawick P., Helmick Beavin J., Don D. Jackson, (1972), Une logique de la communication, trad. Franç. Ed. du Seuil, coll. Points.

\section{NOTES}

1. Voir Gilles Bui-Xuân (1993). Pour une approche psychanalytique du Judo. Quel Corps? Sciences Humaines Cliniques et pratiques corporelles. 45-46, 65-74.

2. Au sens donné par Marcel Mauss aux « techniques du corps ». In : Sociologie et anthropologie, Paris, PUF, 1973.

3. La conation est l'inclination à agir en fonction d'un système de valeurs incorporé. Voir l'Habilitation à Diriger des Recherches de Gilles Bui-Xuân : Pédagogie conative. Le corps mobilisé. Université de Montpellier 1. 1999. Voir également la thèse de JeanPhilippe Turpin : Étude d'enfants abandonniques en situation de judo. Le sens en action. Thèse de Doctorat en STAPS de l'Université de Montpellier 1, 1999. Il montre qu'une discordance socio-conative, qui se repère notamment par le refus du salut, est une entrave majeure à la pratique du combat.

4. Dans Gilles Bui-Xuân (1993). Pour une approche psychanalytique du Judo. Quel Corps? Sciences Humaines Cliniques et pratiques corporelles. 45-46, 65-74, la différence est établie entre un judo de projection dans lequel se joue la mort symbolique de l'adversaire et un judo au sol où il s'agit plutôt de soumission. Or le salut debout ne s'effectue pas de la même manière que le salut au sol, ce dernier étant quelquefois négligé lors des entraînements.

5. D'ailleurs, si le salut des combattants est préservé en compétition, le salut aux juges et arbitres n'est plus exigé, révélant bien qu'il ne constituait qu'une obligation de conformité sans équivalent intériorisé.

6. Voir à ce sujet, Girard R. La Violence et le sacré, Paris, Grasset, 1972.

7. Catherine Kerbrat-Orécchioni est l'auteur de l'article de l'Encyclopaedia universalis, intitulé : sémantique.

8. Voir à ce sujet l'isolement des boxeurs qui peut durer plus d'une semaine avant qu'ils ne réapparaissent. Marcellini A. (1993). Ethnopsychologie sur le ring. Quel Corps?

Sciences Humaines Cliniques et pratiques corporelles. 45-46, 140-153.

9. Voir à ce sujet Baudrillard J. (1976) L'Échange symbolique et la mort. Paris, Gallimard. 


\section{RÉSUMÉS}

Les hommes se sont-ils toujours salués avant de s'affronter en combat singulier ? Et si oui, quelle est la fonction du salut? Référence au divin, conjuration du sort et de la mort, ou simple rappel d'une éthique du combat régulier?

L'étude de trois populations de judokas montre que le rituel du salut est un véritable médium de communication, dont le sens minimum partagé par tous autorise l'entrée dans le combat.

Did men always salute together before fighting in duel? And if yes, what's the salute's function? Reference to the divin, conjuration of fate or death, or simple recall of the ethic in duel according to the rules? Study of three judoka's populations shows that salute's ritual is a real communication's medium, whose minimum meaning, shared by all, allows the entrance into duel.

INDEX

Keywords : rite, judo, communication, salute, ritual, meaning

Mots-clés : salut, sens

\section{AUTEURS}

\section{GILLES BUI-XUÂN}

Faculté des Sciences du Sport et de l'Éducation Physique. Université de Montpellier I. Équipe «Corps et Culture»

\section{SÉBASTIEN RUFFIÉ}

Faculté des Sciences du Sport et de l'Éducation Physique. Université de Montpellier I. Équipe «Corps et Culture » 